staining had prognostic significance that was independent of Gleason grade, using all the cases studied.

PETER FURNESS Department of Pathology, Leicester General Hospital NHS Trust, Gwendolen Road,

Leicester LE5 4PW

1 Sheaff MT, Martin JE, Badenoch DF, Baithun SI. $\beta$ hCG as a prognostic marker in adenocarcinoma of the prostate. $\mathcal{F}$ Clin Pathol 1996; 49:329-32.

Drs Sheaff, Martin and Baithun comment:

We appreciate the interest shown by Dr Furness in our paper. We feel, however, that table 1 may resolve any misunderstandings that may have arisen with regard to Gleason scoring/grading, $\beta$ hCG expression and prognosis. In fact table 1 clearly states the distribution of Gleason grade allocations within the $\beta$ hCG positive group, and it is evident that despite small numbers of cases, even the better differentiated groups had poor clinical outcome and not all metastases or deaths that occurred in this study were in the high grade group. We did perform statistics on the 80 patients as a whole (data not originally presented) and found that $\beta \mathrm{hCG}$ expression and prognosis were closely linked ( $\mathrm{p}<0.002)$. This supports our statement, namely that $\beta$ hCG expression identifies a group of patients with poor prognosis irrespective of histological grade. This does not mean that $\beta \mathrm{hCG}$ expression is entirely independent of histological grading (Gleason grading in particular) or that the latter is not clinically useful, but simply suggests that $\beta$ hCG expression may give valuable additional information when assessing treatment options with regard to further management.

\section{Current guidelines for sampling the cervix in hysterectomy specimens are appropriate}

Guidelines for sampling the cervix in a hysterectomy specimen removed for nonmalignant conditions recommend two midline blocks, one taken from the anterior lip and another from the posterior lip. ${ }^{1-3}$ The purpose of examining these random histological sections is to detect previously unsuspected cervical intraepithelial neoplasia (CIN). In a study examining 100 cervical cone biopsy specimens, taken as part of treatment of CIN, Heatley identified CIN at either the anterior or posterior midline positions (12 and 6 o'clock) in $94 \%$ of cases, with a higher grade of CIN and at a higher frequency than from lateral aspects. ${ }^{4}$

In contrast to Heatley, we assessed the guidelines by examining actual hysterectomy specimens. The hysterectomies had been performed for non-malignant conditions at two centres between February and November 1995. Cervical cytology screening was routine (at two yearly intervals, or more frequently as required) at the Adelaide Women's and Children's Hospital (WCH) but was not at the Universiti Kebangsaan Malaysia (UKM), Kuala Lumpur. Blocks of the cervix were taken from the 12,3,6, and 9 o'clock positions. We identified CIN in one of 72 cases from WCH and three of 40 cases from UKM. CIN was high grade in three cases and low grade in one and was found in the anterior or posterior blocks exclusively in three cases or in either midline block with involvement in a lateral block in one case, but none was found in a lateral block exclusively.

Our study was discontinued at this point as these results verify the findings of Heatley and the current guidelines for sampling of the cervix in hysterectomy specimens.

M M MAYERS

T Y KHONG

Department of Histopathology Women's and Children's Hospital, North Adelaide 5006, Australia

H ABDUL RAHMAN Department of Pathology, Universiti Kebangsaan Malaysia, Kuala Lumpur, Malaysia

1 Rosai J (ed). Guidelines for handling most common and important surgical specimens. In: common and important surgical specimens. In: Ackerman's surgical pathology.
Louis: CV Mosby, 1996:2718.

2 Robboy SJ, Kraus FT, Kurman RJ. Gross description, processing and reporting of gynecologic and obstetric specimens. In: Kurma RJ, ed. Blaustein's pathology of the female genital tract. 3rd edn. Berlin: Springer Verlag, 1987:925-40.

3 Scurry J, Patel K, Wells M. ACP Broadsheet 138: Gross examination of uterine specimens. $\mathcal{F}$ Gross examination of uterine

4 Heatley M. Distribution of cervical intraepithelial neoplasia: are hysterectomy specimens sampled appropriately? F Clin Pathol 1995;48:323 4.

\section{Dr Heatley comments:}

Drs Mayers, Rahman and Khong are to be congratulated for undertaking this study, in two centres in presumably different populations, which confirms the validity of sampling the uterine cervix at the 6 and 12 o'clock positions in hysterectomy specimens. Their results also highlight the continued validity of cervical screening given the lower incidence of CIN lesions in the screened compared with the unscreened population.

\section{Specificity of plasma cell antibody VS38}

In their paper on VS38 staining in melanocytic lesions, Shanks and Banerjee ${ }^{1}$ mention positive staining in a single case of clear cell sarcoma of soft tissue. We have also noted positivity in a wide range of melanocytic lesions with this antibody and have noted strong positivity in a further case of clear cell sarcoma occurring in the vicinity of the ankle joint.

This latter finding is not unexpected as these tumours are regarded as soft part melanomas. However, the situation may be different with other antibodies showing unexpected positivity in malignant melanoma. Although the KP-1 antibody also stains many melanomas, we were previously unable to demonstrate corresponding positivity in any of seven clear cell sarcomas. ${ }^{2}$

We have also noted striking positivity with VS38 in benign schwannoma and would certainly agree that, as with KP-1, VS38 is best used as part of an antibody panel.

K AL-JANABI M CASSIDY Department of Histopathology, Huddersfield Royal Infirmary, Huddersfield HD3 $3 E A$

1 Shanks JH, Banarjee SS. VS38 immunostaining in melanocytic lesions. $\mathcal{f}$ Clin Pathol 1996; 49:205-7.

2 Cassidy M, Loftus B, Whelan A, Sabt B, Hickey $\mathrm{D}$, Henry $\mathrm{K}$, et al. KP-1: not a specific marker.
Staining of 137 sarcomas, 48 Lymphomas, 28 carcinomas, 7 malignant melanomas and 8 cystosarcoma phyllodes. Virchows Arch 1994; 424:635-40

\section{Confidentially, death and the doctor}

The recent article by James and Leadbeatter ${ }^{1}$ raises issues which extend beyond the narrowly medical.

The Tasmanian Court of Appeal in "Pawsey" held by majority that a patient ceases to be a patient at the moment of death. Although this is a Commonwealth case and arose out of Australian Health Insurance Legislation, the Court did not specifically of limit its views of the patient/death issue to the context of that legislation.

Despite views to the contrary, and indeed UK legislation, "Pawsey" may provide a nice point for further argument. In any event, the trial judge's reasons and those of the appeal judges make for a very stimulating read.

J M N HILTON

Director, $\stackrel{\oplus}{\oplus}$

NSW Institute of Forensic Medicine, 42-50 Parramatta Road, 윽 PO Box 90, Glebe, NSW 2037, Australia غ્

1 James DS, Leadbeatter S. Confidentially, death and the doctor. $\mathcal{F}$ Clin Pathol 1996;49:1-4.

\section{Peritoneal involvement by rectal cancer}

Several points emerge from the paper by Shepherd et $a l^{1}$ which deserve comment. The first is the observation that the staging system described by Astler-Coller ${ }^{2}$ and the Australian Clinico-Pathological Staging (ACPS) system $^{3}$ suffer because of their sequential nature as they do not address the possible prognostic influence of local spread beyond the bowel wall in cases where there is lymph node involvement. This is not strictly correct. The most significant contribution made by the Astler-Coller system was to emphasise the importance of direct spread of tumour in 음 cases with lymph node metastases by subclassifying them into stages $\mathrm{C} 1$ and $\mathrm{C} 2$ according 을. to the presence of spread beyond the muscularis propria. Furthermore, when tumour is $\mathcal{N}$ demonstrated histologically in a surgical line $N$ of resection (in practice almost always the $N$ circumferential line) the ACPS system classi- $\omega$ fies the tumour as stage D irrespective of the lymph node status. Thus, local spread may

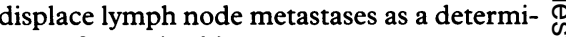
nant of stage in this system.

The second point is that the authors state that there has been little attention to the

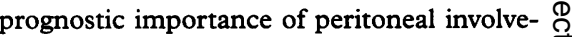
ment in colorectal cancer. In the clinico- $\mathbb{D}$ pathological staging system used in the on-going prospective study of colorectal cancer at Concord Hospital, which began in $\delta$ 1971 , provision is made for separately classifying stage B tumours in which there is direct spread to involve a free serosal surface. ${ }^{4}$ Our experience has been that this substage includes only a small proportion of stage B tumours $(6 \%)$ but is associated with a significantly poorer survival. In a subsequent analysis on patients with stage $C$ tumours, free serosal surface involvement emerged not only as a comparatively common finding (17\%)

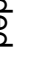

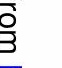

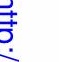

$$
\text { S. }
$$

